\title{
Correction to: Approximation and Online Algorithms
}

\author{
Evripidis Bampis and Nicole Megow
}

\section{Correction to: \\ E. Bampis and N. Megow (Eds.): Approximation \\ and Online Algorithms, LNCS 11926, https://doi.org/10.1007/978-3-030-39479-0}

In the original version of the book, the affiliation of Nicole Megow was wrong. The affiliation has been corrected to:

Department for Mathematics and Computer Science, University of Bremen, Bremen, Germany. 